\title{
Comparação do volume da gota e custo do tratamento dos genéricos de maleato de timolol $0,5 \%$
}

\author{
Drop volume and cost of treatment with generic 0.5\% timolol maleate preparations
}

\author{
João Antonio Prata Junior ${ }^{1}$ \\ João Antonio Prata ${ }^{2}$
}

Faculdade de Medicina do Triângulo Mineiro - Av. Frei Paulino, 30 - Uberaba (MG) CEP 38025-180

${ }^{1}$ Professor Livre-docente do Curso de Pós-graduação em Oftalmologia da Universidade Federal de São Paulo - UNIFESP. Oftalmologista da Faculdade de Medicina do Triângulo Mineiro.

${ }^{2}$ Professor Adjunto, Doutor, Disciplina de Oftalmologia da Faculdade de Medicina do Triângulo Mineiro. Endereço para correspondência: R. Major Eustáquio, 551 - Uberaba (MG) CEP 38010-270

E-mail: jprata@oftalmo.epm.br

Recebido para publicação em 02.07.2003

Versão revisada recebida em 30.02.2004

Aprovação em 06.02.2004

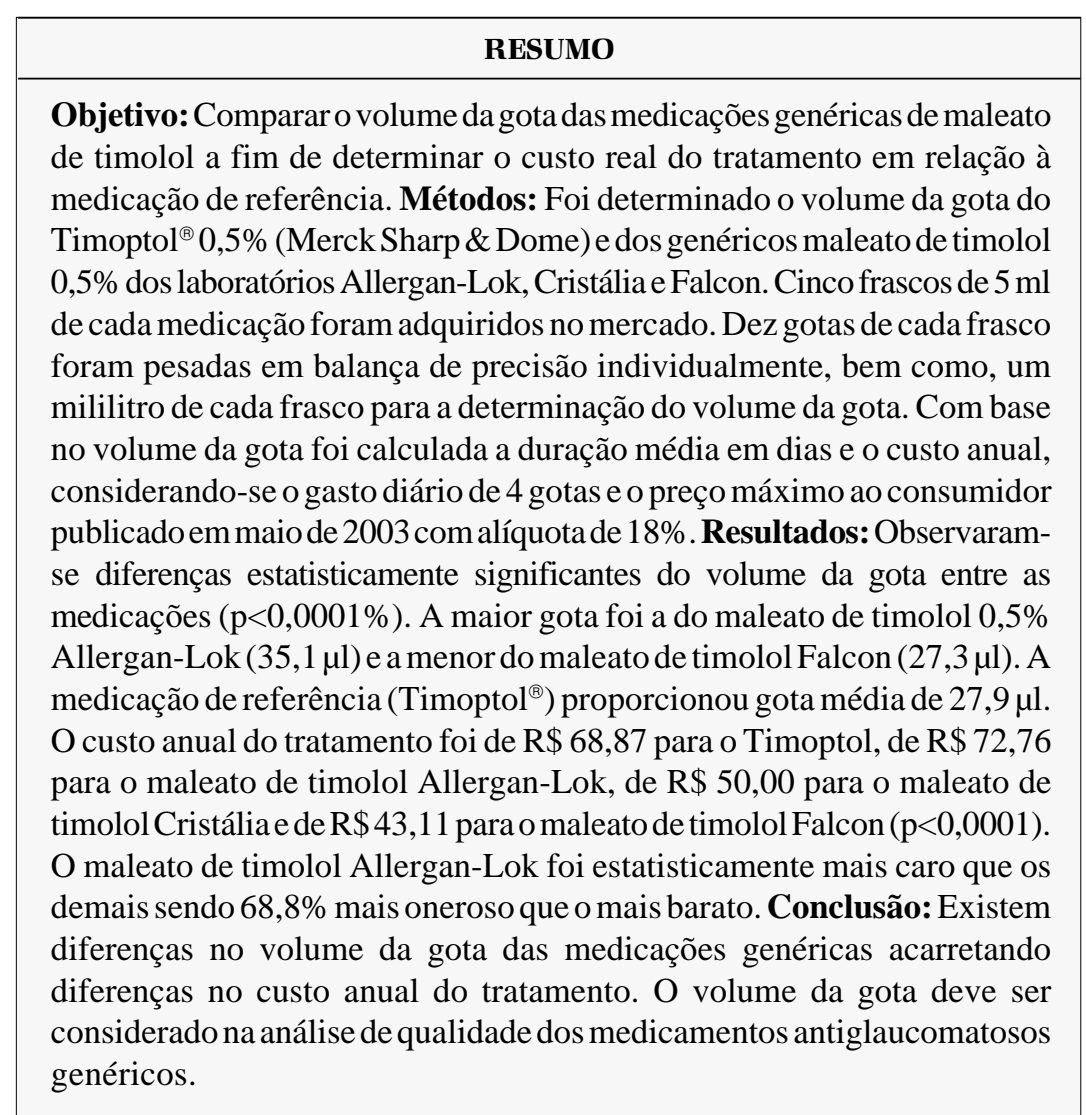

Descritores: Glaucoma/quimioterapia; Glaucoma/economia; Custos de cuidados de saúde; Custos de medicamentos; Soluções oftálmicas/administração \& dosagem; Qualidade dos medicamentos; Fatores socioeconômicos

\section{INTRODUÇÃO}

Os medicamentos genéricos foram recentemente regulamentados no Brasil pela lei $9.787 \mathrm{de} \mathrm{dez} \mathrm{de} \mathrm{fevereiro} \mathrm{de} \mathrm{2000,} \mathrm{com} \mathrm{o} \mathrm{intuito} \mathrm{de} \mathrm{proporcionar}$ acesso a baixo custo a medicações de comprovada eficiência. São formulados a partir de uma apresentação de marca determinada pelo Ministério da Saúde (medicamento referência) e passam por testes de equivalência farmacêutica e bioequivalência a fim de assegurar a sua qualidade ${ }^{(1)}$.

Atualmente são disponíveis medicações genéricas para o tratamento do glaucoma, visando reduzir o custo do tratamento da doença, que no Brasil é considerável ${ }^{(2-4)}$. Pedroso et al salientam que aproximadamente $13 \%$ do orçamento familiar fica comprometido com o tratamento do glaucoma ${ }^{(3)}$. Já 
Silva et al referem um percentual ainda mais elevado $(15,5 \%)$, sendo que em sua série, aproximadamente $24 \%$ dos pacientes tiveram $25 \%$ ou mais de sua renda destinada ao tratamento antiglaucomatoso ${ }^{(4)}$.

Vários fatores estão envolvidos nos custos da terapia antiglaucomatosa. $^{(2-4)}$ Dentre elas, destaca-se o tamanho da gota proporcionada pelo frasco da medicação ${ }^{(5-10)}$. Para que sejam evitados desperdícios, a gota ideal deve ter um volume entre 20 a $30 \mu$, tendo em vista o reduzido volume que o saco conjuntival comporta ${ }^{(5-10)}$.

Em publicação recente, foram detectadas diferenças entre o tamanho da gota de apresentações nacionais e norte-americanas da mesma droga e do mesmo fabricante ${ }^{(10)}$. Isto se associa a um custo maior do tratamento com as apresentações nacionais, chegando em alguns casos a ser da ordem de $30 \%$. Este fato ocasionou que alguns fabricantes modificassem os frascos das apresentações nacionais, visando minimizar esta diferença.

As medicações genéricas têm por princípio um menor preço ao consumidor que a de referência. Entretanto, não há dados se o menor custo do frasco de colírio genérico realmente proporciona uma economia no tratamento, tendo em vista que o tamanho da gota das suas apresentações ainda não foi comparado.

Este estudo tem por objetivo comparar o volume da gota das medicações genéricas de maleato de timolol a fim de determinar o custo real do tratamento em relação à medicação de referência.

\section{MÉTODOS}

Foram adquiridos no mercado da cidade de Uberaba cinco frascos de cada apresentação genérica e da de referência em abril de 2003. As medicações testadas foram o Timoptol ${ }^{\circledR} 0,5 \%$ (Merck Sharp \& Dome, referência dos genéricos), maleato de timolol 0,5\% (Allergan Lok), maleato de timolol 0,5\% (Cristália) e o maleato de timolol 0,5\% (Falcon), todos com $5 \mathrm{ml}$. O maleato de timolol $0,5 \%$ da Apotex não foi avaliado, pois não foi encontrado no mercado. O laboratório Apotex foi contatado através do seu sistema de atendimento ao consumidor que informou que o maleato de timolol realmente estava em falta no mercado por dificuldade de importação do princípio ativo.

Para determinação do volume da gota procedeu-se a pesagem de 10 gotas de cada frasco usando-se balança digital de precisão da marca Sartorius devidamente calibrada, que permitia mensurar até quatro casas decimais de grama (Figura 1). Após a medida de cada gota, a balança era zerada e cinco medidas eram realizadas segurando-se o frasco com a mão direita e cinco com a mão esquerda (Figura 1). Foi também pesado um mililitro de cada frasco, mensurado com o uso de uma pipeta de um mililitro. Desta forma, o volume da gota foi determinado pela proporção entre o peso de um mililitro e o peso da gota.

De posse do volume da gota foi determinado, para cada frasco, o número total de gotas disponíveis pela relação com o

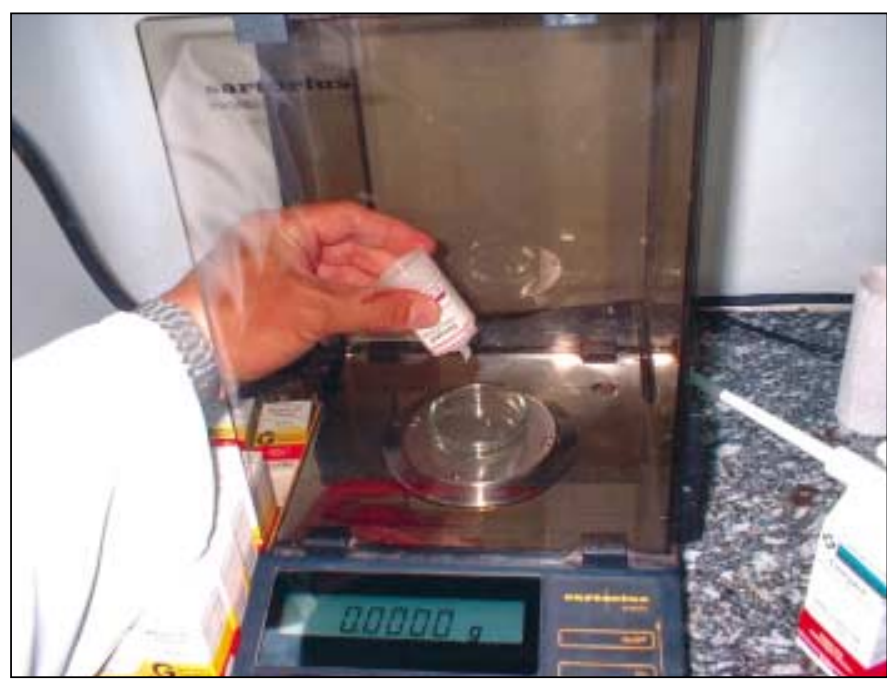

Figura 1 - Pesagem da gota (mão esquerda)

volume do frasco expresso no seu rótulo. Considerando-se a posologia de quatro gotas diárias (duas por olho) sem desperdícios calculou-se o custo da gota, o número total de frascos necessários para o tratamento de um ano e o custo anual do tratamento. O preço avaliado para cada medicação foi o máximo ao consumidor com alíquota de $18 \%$ publicado para o mês de maio de 2003, ou seja, $\mathrm{R} \$ 8,44$ para o Timoptol ${ }^{\circledR}, \mathrm{R} \$ 7,10$ para o maleato de timolol Allergan, $\mathrm{R} \$ 5,66$ para o maleato de timolol Cristália e R \$ 5,41 para maleato de timolol Falcon.

Os dados foram tabelados e analisados estatisticamente por análise de variância (ANOVA). Para comparação entre as medicações foram realizados testes de comparações múltiplas (TukeyKramer). Considerou-se significância estatística p $<0,05$.

O protocolo do estudo foi submetido à Comissão de Ética em Pesquisa da Faculdade de Medicina do Triângulo Mineiro (número 371/2003).

\section{RESULTADOS}

A tabela 1 expressa o volume médio da gota determinado para cada tipo de medicação testada, a sua duração média em dias (frascos de $5 \mathrm{ml}$ ) e o custo anual de cada medicação.

A comparação do volume da gota por análise de variância (ANOVA) apresentou significância estatística $(\mathrm{p}<0,0001)$. A

\begin{tabular}{|c|c|c|c|}
\hline Medicação & $\begin{array}{c}\text { Volume gota } \\
(\mu \mathrm{I})\end{array}$ & $\begin{array}{l}\text { Duração } \\
\text { (dias) }\end{array}$ & $\begin{array}{c}\text { Custo anual } \\
\text { (R\$) }\end{array}$ \\
\hline Timoptol $^{\circledR}$ & $27,9 \pm 0,4$ & $44,7 \pm 0,7$ & $68,90 \pm 1,1$ \\
\hline Allergan & $35,1 \pm 0,8$ & $35,6 \pm 0,8$ & $72,76 \pm 1,7$ \\
\hline Cristália & $30,2 \pm 2,7$ & $41,6 \pm 3,8$ & $50,00 \pm 4,5$ \\
\hline Falcon & $27,3 \pm 0,5$ & $45,8 \pm 0,9$ & $43,11 \pm 0,8$ \\
\hline $\mathrm{p}$ & $<0,0001$ & $<0,0001$ & $<0,0001$ \\
\hline
\end{tabular}


menor gota detectada foi a do maleato de timolol Falcon $(27,3 \pm 0,5 \mu \mathrm{l})$ e a maior a do maleato de timolol Allergan-Lok $(35,1 \pm 0,8 \mu \mathrm{l})$. O teste de comparações múltiplas mostrou que o volume da gota do maleato de timolol 0,5\% Allergan-Lok diferiu estatisticamente de todos os demais $(\mathrm{p}<0,001)$. Não foi observada diferença estatisticamente significante entre o volume da gota do Timoptol ${ }^{\circledR}$ e o maleato de timolol Cristália, bem como, quando comparada ao do maleato de timolol Falcon. A comparação entre o maleato de timolol Falcon e o Cristália foi estatisticamente significante $(\mathrm{p}<0,05)$.

A comparação da duração em dias de cada medicação por análise de variância (ANOVA) apresentou significância estatística ( $\mathrm{p}<0,0001)$. A menor duração detectada foi a do maleato de timolol Allergan-Lok $(35,6 \pm 0,8$ dias $)$ e a maior a do maleato de timolol Falcon $(45,8 \pm 0,9$ dias). O teste de comparações múltiplas mostrou que a duração média do maleato de timolol 0,5\% Allergan-Lok diferiu estatisticamente de todos os demais (p Allergan-Lok x Timoptol ${ }^{\circledR}=$ p Allergan-Lok x Falcon $<0,001$; Allergan-Lok x Cristália $\mathrm{p}<0,01)$. Não foi observada diferença estatisticamente significante entre a duração do Timoptol $^{\circledR}$ e o maleato de timolol Cristália, bem como, quando comparada ao do maleato de timolol Falcon. A comparação entre o maleato de timolol Falcon e o Cristália foi estatisticamente significante $(\mathrm{p}<0,05)$.

A comparação do custo anual do tratamento com cada medicação mostrou significância estatística pela análise de variância (ANOVA) $(\mathrm{p}<0,0001)$. O maior custo foi do maleato de timolol Allergan-Lok $(\mathrm{R} \$ 72,76 \pm 1,7)$ e o menor do maleato de timolol Falcon $(\mathrm{R} \$ 43,11 \pm 0,8)$. O teste de comparações múltiplas mostrou que não houve diferença estatística entre o Timoptol $^{\circledR}$ e o maleato de timolol Allergan-Lok. O custo do maleato de timolol Falcon foi estatisticamente menor que todos os demais ( $\mathrm{p}<0,001$ para Falcon $\mathrm{x}$ Allergan-Lok e Falcon $\mathrm{x}$ Timoptol ${ }^{\circledR}$; Cristália x Falcon $\left.\mathrm{p}<0,01\right)$.

\section{DISCUSS $\tilde{A} O$}

Os resultados deste estudo são concordantes com estudos anteriormente publicados que ressaltam o importante papel do tamanho da gota de antiglaucomatosos tópicos no custo do tratamento ${ }^{(5-10)}$.

A comparação estatística do custo anual do tratamento demonstrou que a medicação genérica maleato de timolol Allergan-Lok está associada a um custo maior em relação aos outros genéricos testados devido ao fato de que seu frasco proporciona a maior gota estudada nesta série. Quando comparada à medicação de referência, observou-se um maior valor do custo anual, porém sem significância estatística. Ou seja, o genérico maleato de timolol Allergan-Lok associa-se a um custo anual similar à medicação de referência Timoptol ${ }^{\circledR}$. Tais achados carecem de precedência na literatura e indicam que o volume da gota deva ser incluído no controle de qualidade das medicações genéricas para glaucoma.

Considerando-se a comparação entre os genéricos observa- se que o maleato de timolol Allergan-Lok está associado a um custo anual $68,8 \%$ maior que o genérico de custo anual menor (maleato de timolol Falcon). Isto tem grande importância social levando-se em conta o baixo poder aquisitivo da população brasileira que segundo Pedroso et al gasta anualmente $13 \%$ do orçamento familiar no tratamento do glaucoma ${ }^{(3)}$.

Deve-se ressaltar que a maioria das medicações testadas apresentaram volume de gota dentro da faixa considerada ideal (20-30 $\mu \mathrm{l})^{(5-10)}$, com exceção da maleato de timolol AllerganLok, cujo volume ligeiramente superior $(35,1 \pm 0,8 \mu \mathrm{l})$.

\section{CONCLUS ÃO}

Os resultados deste estudo demonstram que existem diferenças no volume da gota das medicações genéricas acarretando diferenças no custo anual do tratamento.

\section{AGRADECIMENTOS}

Os autores agradecem ao laboratório de patologia especial da Faculdade de Medicina do Triângulo Mineiro por permitir o uso dos equipamentos para o experimento.

\section{ABSTRACT}

Purpose: To compare drop volume and treatment cost of generic $0.5 \%$ timolol maleate preparations. Methods: The drop volume of Timoptol ${ }^{\circledR} 0.5 \%$ (Merck Sharp \& Dohme) and its generic preparations (Allergan-Lok, Cristália and Falcon) were determined. Five bottles of each medication were purchased at local drugstores. Using a precision scale, ten drops and one mililiter of each bottle were weighed. Drop volume was calculated by the relation between volume and weight. Duration, in days, of each bottle and annual cost were calculated considering a daily use of 4 drops and maximum price to consumers published May, 2003. Results: Statistically significant differences were observed between drop volumes $(\mathrm{p}<0.0001 \%)$. Allergan-Lok timolol maleate had the largest drop $(35.1 \mu \mathrm{l})$ and the smallest was Falcon timolol maleate $(27.3 \mu \mathrm{l})$. Timoptol ${ }^{\circledR}$ drop volume was $27.9 \mu \mathrm{l}$. Annual treatment costs were $\mathrm{R} \$ 68.87$ for Timoptol, R \$ 72.76 for Allergan-Lok timolol maleate, R \$ 50.00 for Cristalia timolol maleate and R \$ 43.11 for Falcon timolol maleate $(\mathrm{p}<0.0001)$. Allergan-Lok timolol maleate was statiscally more expensive than the others and it was $68.8 \%$ more expensive than the cheapest. Conclusion: There are differences in drop volume among generic medications for glaucoma which are related to their annual cost. Drop volume should be considered in the analysis of quality of generic preparations.

Keywords: Glaucoma/drug therapy; Glaucoma/economics; Health care costs; Drug costs; Ophthalmic solutions/administration \& dosage; Drug quality; Socioeconomic factors 


\section{REFERÊNCIAS}

1. Conselho Regional de Medicina de São Paulo. Medicamentos genéricos. São Paulo: Assessoria de Imprensa do CRF-SP e Cremesp; 2001. p.109.

2. Prata Jr JA, Vita Sobrinho JB. Tratamento clínico do glaucoma. In: Vita Sobrinho JB, editor. Farmacologia terapêutica. Rio de Janeiro: Cultura Médica; 1999. p.272-95.

3. Pedroso L, Carvalho Jr ES, Paranhos Jr A, Prata Jr JA, Mello PAA. Custo real do tratamento do glaucoma para o paciente. Arq Bras Oftalmol 1999;62:677-82.

4. Silva LMS, Vasconcelos JPC, Temporini ER, Costa VP, Kara-José N. Tratamento clínico do glaucoma em um hospital universitário: custo mensal e impacto na renda familiar. Arq Bras Oftalmol 2002;65:299-303.

5. Lederer CM, Harold RE. - Drop size of commercial glaucoma medications. Am J Ophthalmol 1986;101:691-4.
6. Stewart WC, Sine C, Cate E, Minno GE, Hunt HH. Daily cost of betaadrenergic blocker therapy [commented on Arch Ophthalmol 1997;115:914]. Arch Ophthalmol 1997;115:853-6.

7. Fiscella RG, Geller JL, Gryz L, Willensky J, Viana M. Cost considerations of medical therapy for glaucoma. Am J Ophthalmol 1999;128:426-33.

8. Brown RH, Hotchkiss ML, Davis B. Creating smaller eyedrops by reducing eyedropper tip dimensions. Am J Ophthalmol 1985;99:460-4.

9. Vaidergorn PG, Susanna Jr R, Borges AS, Giampani Jr J. Tempo de terapêutica propiciado por frasco de colírios hipotensores oculares. Rev Bras Oftalmol 2002;61:278-82.

10. Roizenblatt R, Freitas D, Belfort Jr R, Höfling-Lima AL, Prata Jr.JA. Impacto econômico no tratamento do glaucoma: volume de gotas de colírios brasileiros e norte americanos. Arq Bras Oftalmol 2001;64:143-6.

\title{
XL REUNIÃO DO CENTRO DE ESTUDOS OFTALMOLÓGICOS "CYRO DE REZENDE"
}

\author{
02 a 04 de Dezembro de 2004 \\ Centro de Convenções de Ribeirão Preto - SP
}

Departamento de 0 ftalmologia, 0 torrinolaringologia e Cirurgia de Cabeça e Pescoço da Faculdade de Medicina da USP de Ribeirão Preto

Tema: "Superfície 0 cular"

INFORMAÇÕES: Eventus Planejamento e Organização

Fone: (11) 3361-3056 - Fax: (11) 3361-3089

E-mail: oftalmo@eventus.com.br

Home-page: www.eventus.com.br/ oftalmo 